\title{
17DD Yellow Fever Revaccination and Heightened Long-Term Immunity in Populations of Disease-Endemic Areas, Brazil
}

Ana Carolina Campi-Azevedo, Vanessa Peruhype-Magalhāes, Jordana Grazziela Coelho-dos-Reis, Lis Ribeiro Antonelli, Christiane Costa-Pereira, Elaine Speziali, Laise Rodrigues Reis, Jandira Aparecida Lemos, José Geraldo Leite Ribeiro, Luiz Antônio Bastos Camacho, Maria de Lourdes de Sousa Maia, Sheila Maria Barbosa de Lima, Marisol Simões, Reinaldo de Menezes Martins, Akira Homma, Luiz Cosme Cota Malaquias, Pedro Luiz Tauil,

Pedro Fernando Costa Vasconcelos, Alessandro Pecego Martins Romano, Carla Magda Domingues, Andréa Teixeira-Carvalho, Olindo Assis Martins-Filho; Collaborative Group for Studies of Yellow Fever Vaccine

We evaluated the duration of neutralizing antibodies and the status of 17DD vaccine-specific T- and B-cell memory following primary and revaccination regimens for yellow fever (YF) in Brazil. We observed progressive decline of plaquereduction neutralization test (PRNT) seropositivity and of the levels of effector memory CD4+ and CD8+ T cells, as well as interferon $-\gamma+C D 8+T$ cells, 10 years after primary vaccination. Revaccination restored PRNT seropositivity as well as the levels of effector memory CD4+, CD8+, and interferon$\gamma+$ CD8 + T cells. Moreover, secondary or multiple vaccinations guarantee long-term persistence of PRNT positivity and cell-mediated memory 10 years after booster vaccination.

Author affiliations: Instituto René Rachou of Fundação Oswaldo Cruz (FIOCRUZ-Minas), Belo Horizonte, Brazil (A.C. Campi-Azevedo,

V. Peruhype-Magalhāes, J.G. Coelho-dos-Reis, L.R. Antonelli,

C. Costa-Pereira, E. Speziali, L.R. Reis, A. Teixeira-Carvalho, O.A. Martins-Filho); Universidade Federal de Minas Gerais, Belo Horizonte (J.G. Coelho-dos-Reis); Secretaria Municipal de Saúde, Belo Horizonte (J.A. Lemos); Secretaria do Estado de Saúde de Minas Gerais, Belo Horizonte (J.G.L. Ribeiro); Escola Nacional de Saúde Pública (FIOCRUZ-Rio), Rio de Janeiro, Brazil (L.A.B. Camacho); Instituto de Tecnologia em Imunobiológicos Bio-Manguinhos (FIOCRUZ-Rio), Rio de Janeiro (M. de Lourdes de Sousa Maia, S.M. Barbosa de Lima, M. Simões,

R. de Menezes Martins, A. Homma); Universidade Federal de Alfenas, Alfenas, Brazil (L.C.C. Malaquias); Universidade de Brasília, Brasília, Brazil (P.L. Tauil); Instituto Evandro Chagas, Ananindeua, Brazil (P.F.C. Vasconcelos); Secretaria de Vigilância em Saúde-Ministério da Saúde, Brasília (A.P.M. Romano,

C.M. Domingues)

DOI: https://doi.org/10.3201/eid2508.181432
These findings support the relevance of booster doses to heighten the 17DD-YF-specific immune response to guarantee the long-term persistence of memory components. Secondary or multiple vaccinations improved the correlates of protection triggered by 17DD-YF primary vaccination, indicating that booster regimens are needed to achieve efficient immunity in areas with high risk for virus transmission.

Y ellow fever (YF) vaccination is recommended for persons living in YF-endemic areas as the most effective strategy to reduce the risk for infection (1). The 17D and 17DD live attenuated vaccines are considered similarly safe and immunogenic, regardless of the minor differences in their nucleotide sequences (1). The progressive expansion of areas with YF viral circulation in YF-endemic countries has required extensive vaccination campaigns that reduced the international vaccine stockpile and brought to light the discussion about the need for booster doses to guarantee long-term cell memory in populations living in YF-endemic countries. Outbreaks of YF occur occasionally in areas of Africa and South America (2-7).

In 2013, the World Health Organization (WHO) stated that a single dose of YF vaccine sufficed to provide lifelong protection and that no booster dose was required to guarantee protection against the disease $(1,8)$. However, time-dependent loss of protective immunity has been reported (9-11). The levels of YF-neutralizing antibodies decrease significantly 10 years after vaccination; $\approx 25 \%-30 \%$ of primary vaccinees lack protective antibodies $(10,11)$. In addition, the polyfunctional cellular immune responses elicited by YF vaccination that contribute to protection also 
displayed a time-dependent decline following primary vaccination $(11,12)$. In light of this information, the single-dose regimen for $\mathrm{YF}$ vaccine has been questioned, especially in YF-endemic countries where the proportion of persons exposed to potential risks should be considered against the primary-vaccine failure rate and time-dependent decline of protective immunity.

The goal of this study was to evaluate the proxies of protection elicited by primary, secondary, and multiple vaccinations and verify the duration of neutralizing antibodies and 17DD-specific T- and B-cell memory following these distinct vaccination regimens. We sought to clarify the importance of 17DD-YF booster vaccination to heighten the immune response of those primary vaccinees living in endemic areas whose immunity declines to nonprotective levels.

\section{Materials and Methods}

\section{Study Population}

We conducted this investigation during May 12, 2014-December 16, 2016, simultaneously sampling from Rio de Janeiro and 2 municipalities of Minas Gerais state (Alfenas and Ribeirão das Neves), Brazil. We assigned participants to groups on the basis of official vaccination records. The study included 421 samples collected from 326 healthy adults $18-77$ years of age, initially categorized into 3 arms: primary vaccination, secondary vaccination, and multiple vaccination (Figure 1). We designed the primary vaccination and secondary vaccination arms as 2 complementary independent approaches, each including a longitudinal (95 paired samples) and a cross-sectional investigation (231 unpaired samples). Study groups were coded to indicate participants' vaccination status (NV for nonvaccinated persons, PV for those who had had primary YF vaccination only, RV for those who had been revaccinated) and time since last vaccination, given in days or years (e.g, d0 for day zero).

\section{Samples and Tests}

We collected whole blood samples from each participant. We used samples of $5 \mathrm{~mL}$ without anticoagulant for plaque-reduction neutralization test (PRNT) and samples of $20 \mathrm{~mL}$ in heparin for 17DD-YF phenotypic and functional analyses.

\section{PRNT}

We used serum samples to quantify the PRNT levels to the 17DD-YF virus by the micro-PRNT50 test, as described previously by Simões et al. (13). We performed assays at Laboratório de Tecnologia Virológica (LATEV), BioManguinhos, and expressed results from replicates as the reciprocal of sample dilution, considering seropositivity of PRNT titers $>1: 50$ serum dilution.

\section{Dengue IgG Indirect ELISA}

We performed serologic tests for dengue virus (DENV) IgG using a Panbio dengue IgG indirect ELISA kit (https://www.alere.com). Tests were performed at Laboratório de Flavivírus, Instituto Osvaldo Cruz, as previously reported (14).

\section{Phenotypic and Functional Memory Biomarkers}

We performed in vitro 17DD-YF-specific peripheral blood lymph proliferative assay as previously reported by Costa-Pereira et al. (12). In brief, we incubated replicates of PBMC suspension $\left(1.0 \times 10^{6} /\right.$ well $)$ for 144 hours at $37^{\circ} \mathrm{C}$ in $5 \% \mathrm{CO}_{2}$. We harvested cells from control (CC) and 17DDYF antigen-stimulated (17DD-YF Ag) cultures, labeled them with live/dead dye (Life Technologies, https://www. thermofisher.com), and used a cocktail of monoclonal antibodies (mAbs) to quantify the phenotypic memory status of $\mathrm{T}$ cells and B cells. For T cells we used anti-CD4/(RPAT4)/FITC, anti-CD8/(SK1)/PerCP-Cy5.5, anti-CD27/(MT271)/PE, anti-CD45RO/(UCHL1)/PE-Cy7, and anti-CD3/

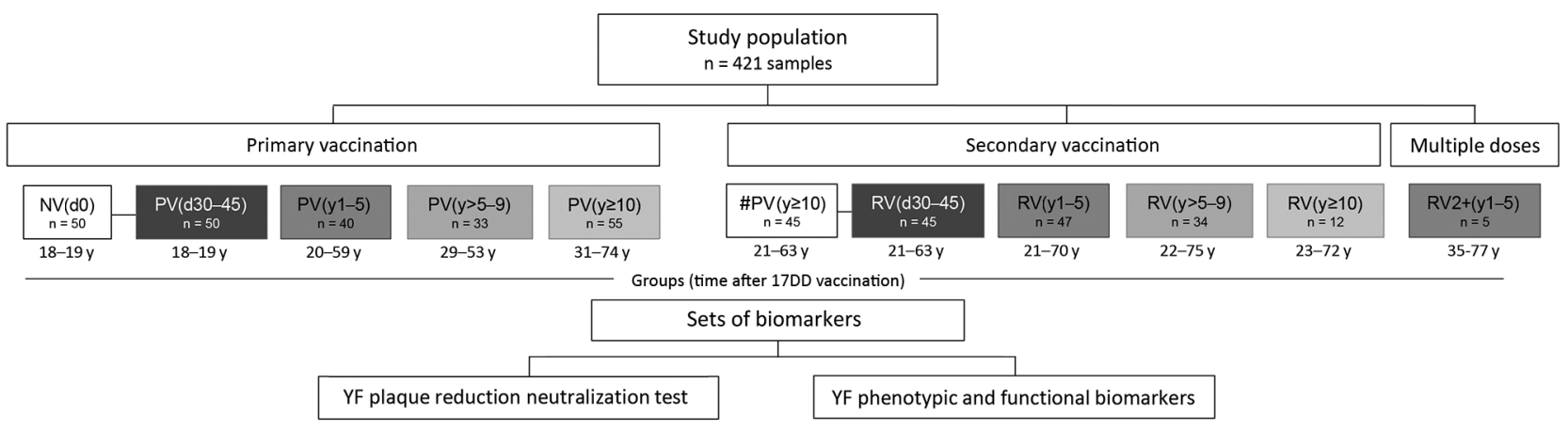

Figure 1. Study population and methods for analyzing 17DD vaccine-specific neutralizing antibodies and phenotypic/functional cell memory in YF. The primary vaccination arm (reference group) includes participants who have never been vaccinated or have had 1 YF vaccination; secondary vaccination arm includes participants who have received 1 or 2 vaccinations; and multiple doses arm includes participants who have received $\geq 2$ revaccinations. Participant subgroups indicate number of days or years since vaccination (in parentheses; $\mathrm{d} 0$ for those never vaccinated). Participant age ranges are given below subgroup boxes. \#PV, had primary vaccination $\geq 10$ years previously; NV, not vaccinated; PV, had primary vaccination only; RV, revaccinated; YF, yellow fever. 
(SK7)/APC-Cy7; for B cells, we used anti-CD19/(HIB19)/ PerCP, anti-CD27/(M-T271)/PE, and anti-IgD/(IA6-2)/ FITC. We obtained all mAbs from BD Pharmingen (https:// bdbiosciences.com).

In parallel, we stained cultured PBMC aliquots with live/dead dye and a mix of mAbs to quantify the functional memory status of T and B cells: anti-CD3/(UCHT1)/ Qdot605 (Invitrogen, https://www.thermofisher.com); antiCD4/(GK1.5)/APCe-Fluor780 (eBioscience, https://www. thermofisher.com); anti-CD8/(SK1)/PerCP (BD Biosciences, https://bdbiosciences.com); and anti-CD19/(HIB19)/ Alexa-Fluor700 (eBioscience). After a fix/permeabilize procedure, we incubated cells with a mAbs cocktail of antiTNF- $\alpha /($ clone MAb11)/PE-Cy7, anti-interferon (IFN)- $\gamma /$ (clone B27)/Alexa-Fluor488), anti-interleukin (IL)-5/ (JES1-39D10)/PE, and anti-IL-10/(JES3-19F1)/APC, all from BD Biosciences. We fixed the stained cells and stored them at $4^{\circ} \mathrm{C}$ for $\leq 24$ hours before acquisition on a BD LSR Fortessa Flow Cytometer (BD Biosciences).

We acquired a total of 100,000 lymphocytes from each sample. We used FlowJo version 9.3.2 software (Tree Star, https://www.flowjo.com) to quantify the memory Tcell and B-cell subsets, as well as the percentage of cytokine-producing $\mathrm{T}$ and $\mathrm{B}$ cells. We expressed the results as $17 \mathrm{DD}-\mathrm{YF} \mathrm{Ag} / \mathrm{CC}$ Index, calculated as the ratio of cells observed in the 17DD-YF Ag cultures divided by the respective control culture.

\section{Data Analysis}

This study was composed of 2 independent but complementary approaches: a longitudinal investigation and a cross-sectional investigation. We performed statistical analyses for the longitudinal investigation using paired t-test to compare $\mathrm{NV}(\mathrm{d} 0)$ versus $\mathrm{PV}(\mathrm{d} 30-45)$ groups for primary vaccination, as well as $\# \mathrm{PV}(\mathrm{y} \geq 10)$ (primary vaccination $>10$ years ago) versus $\mathrm{RV}(\mathrm{d} 30-45)$ for secondary vaccination. For the cross-sectional design, we made the transversal comparisons among groups using analysis of variance adjusted to multiple comparisons and set statistical significance at $\mathrm{p}<0.05$. (We did not highlight nonsignificant differences in the figures.) We used the $\chi^{2}$ test to compare seropositivity rates between NV and PV groups and also between \#PV and RV groups.

We performed biomarker signature analysis as described previously by Luiza-Silva et al. (15). In brief, we calculated the global median value of 17DD-YF Ag/CC Index for each phenotypic and functional biomarker and used that value as the cutoff to identify each biomarker as low index (below global median) or high index (above global median). We considered only biomarkers observed in $>50 \%$ of study participants for comparative analysis among groups.

We conducted Venn diagram analysis (http://bioinformatics.psb.ugent.be/webtools/Venn) to select common biomarkers among subgroups. We overlaid biomarker signatures for comparative analysis of time-dependent changes of biomarker sets observed after vaccination.

\section{Results}

\section{Booster Vaccination and PRNT}

Data analysis demonstrated that primary vaccination triggered significant levels of 17DD-YF-specific neutralizing antibodies $(\mathrm{p}<0.0001)$, reaching a seropositivity rate of $96 \%$ (Figure 2). Of note, we observed progressive decrease in PRNT levels $(p<0.0001)$ and in the PRNT seropositivity rates along the time compared with $\mathrm{PV}(\mathrm{d} 30-45)$. The seropositivity rate declined to $\approx 71 \%$ by 10 years after
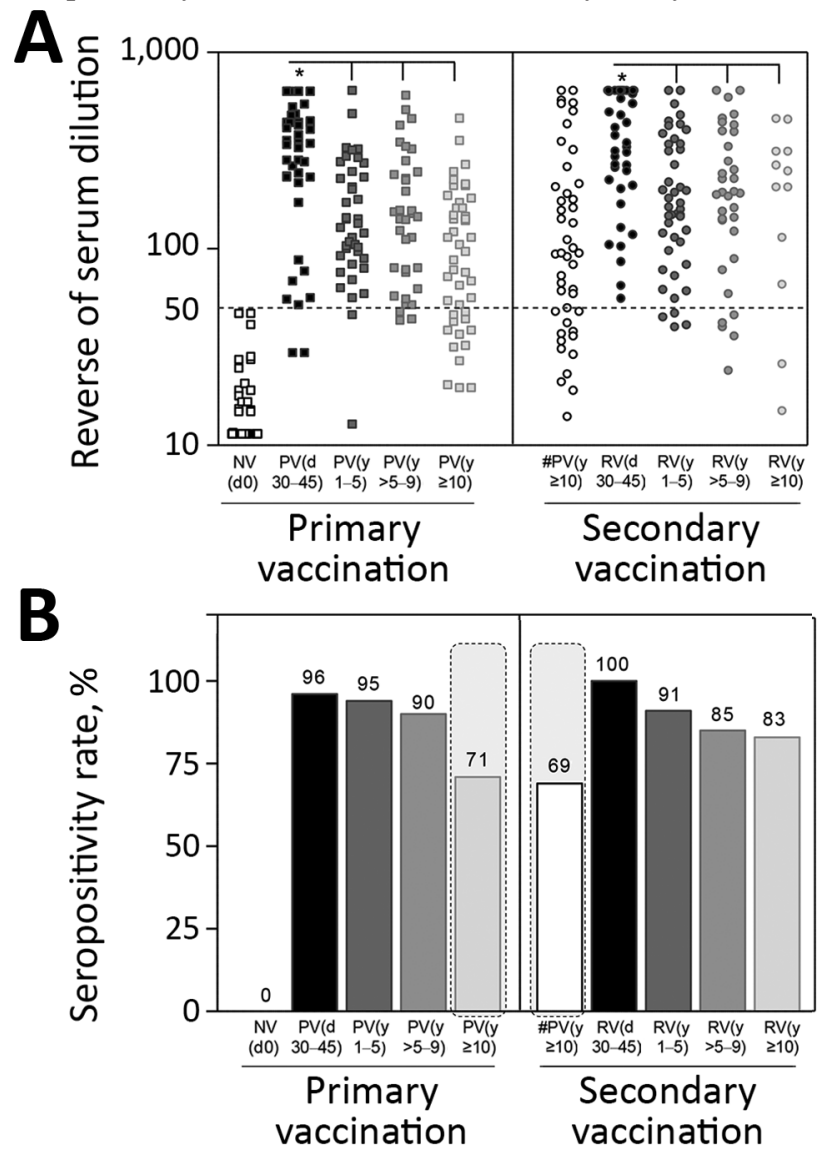

Figure 2. Neutralizing antibody levels and seropositivity rates before and after primary and secondary 17DD vaccination for YF. We detected 17DD-specific neutralizing antibodies by micro plaque-reducing neutralization test (micro-PRNT50) and determined seropositivity rates by considering serum dilution $>1: 50$ as the cutoff criterion for PRNT positivity. A) Scatter graph of PRNT titers, expressed as reverse of serum dilution. B) Percentage of PRNT seropositivity (serum dilution $>1: 50$ ). Gray dashed lines indicate critical seropositivity rates $<80 \%$. Participant subgroups indicate number of days or years since vaccination (in parentheses; d0 for those never vaccinated). \#PV, had primary vaccination $\geq 10$ years previously; NV, not vaccinated; PV, had primary vaccination only; $\mathrm{RV}$, revaccinated; $\mathrm{YF}$, yellow fever. 
primary vaccination (Figure 2, panel B). Booster vaccination significantly increased the PRNT levels $(\mathrm{p}<0.0001)$ and raised the seropositivity rate from $69 \%$ to $100 \%$ (Figure 2, panels A, B). The secondary vaccination was accompanied by higher seropositivity rate after 10 years upon booster dose, regardless of the decrease in PRNT levels $(p<0.0001)$ observed over time compared with \#PV(d30-45) (Figure 2, panel B).

\section{Secondary Booster Vaccination and YF-specific Cell-Mediated Memory}

Comparative analysis of $\mathrm{NV}(\mathrm{d} 0)$ versus $\mathrm{PV}(\mathrm{d} 30-45)$ after 1 or 2 doses of 17DD-YF vaccine demonstrated that primary vaccination is followed by an increase of memory $\mathrm{T}$ cells, including eEfCD4 $(p<0.05)$, EMCD4 $(p<0.05)$, and EMCD8 $(p=0.0006)$, and all B-cell subsets evaluated, $\operatorname{NCD} 19(\mathrm{p}=0.01), \mathrm{nCMCD} 19(\mathrm{p}=0.001)$, and CMCD19 $(p=0.001)$. We also observed a decrease of NCD8 ( $p=$ $0.02)$, eEfCD8 $(p=0.02)$, and CMCD8 $(p=0.001)$. The results showed that cellular immunity, eEfCD4 $(p=0.01)$, EMCD4 $(p=0.01)$, and EMCD8 $(p=0.009)$; and all B-cell subsets, NCD19 ( $\mathrm{p}=0.003), \mathrm{nCMCD} 19(\mathrm{p}=0.02)$, and CMCD19 $(p=0.0002)$, clearly wane over 10 years, compared with 30-45 days after primary vaccination (Figure 3, https://wwwnc.cdc.gov/EID/article/25/8/18-1432-F3.htm).

Secondary vaccination with $17 \mathrm{DD}-\mathrm{YF}$ was able not only to increase the level of memory T-cell subsets, eEfCD4 ( $<<0.05)$, EMCD4 $(\mathrm{p}<0.05)$, and EMCD8 ( $\mathrm{p}=$ 0.04 ) at $30-45$ days after the second dose but also to sustain the maintenance of eEfCD4 and EMCD8 levels even after $\geq 10$ years upon booster vaccination compared with 30-45 days after secondary vaccination. We observed no substantial changes in memory B-cell subsets upon secondary vaccination (Figure 3 ).

\section{Revaccination and IFN- $\gamma$-Mediated T-Cell Memory}

Data analysis for in vitro 17DD-YF antigen recall revealed that primary vaccination induced significant increases of functional memory biomarkers in CD4+ (tumor necrosis factor $[\mathrm{TNF}]-\alpha / \mathrm{p}=0.04$, IFN- $-\gamma / \mathrm{p}=0.04$ and IL-5/p $=$ $0.0001)$ and CD $8+\mathrm{T}$ cells $(\mathrm{TNF}-\alpha / \mathrm{p}=0.0003, \mathrm{IFN}-\gamma / \mathrm{p}=$ 0.008 and IL-5/p $=0.0002$ ) as well as in B cells (TNF- $\alpha /$ $\mathrm{p}<0.05$ and IL-5/p $=0.016$ ) (Figure 4). We observed a decrease of IL-10+CD4+ T cells $(p=0.04)$ at $30-45$ days after primary vaccination and a clear decrease of TNF- $\alpha$, IFN- $\gamma$, and IL- 5 produced by CD4+ and CD $8+$ T cells. We also saw a decrease in TNF- $\alpha$ and IL- 5 from B cells over time, particularly at $>10$ years, compared with 30-45 days after primary vaccination ( $\mathrm{p}<0.05$ in all cases). Conversely, we detected an increase of IL-10+ B cells over time after primary vaccination (Figure 4 ).

Secondary vaccination was able to restore T-cell functional memory mediated by IFN- $\gamma$ and B-cell functional memory by TNF- $\alpha$. We observed sustained production of IFN- $\gamma$ by CD $8+T$ cells even $\geq 10$ years after booster vaccination (Figure 4).

\section{Booster Vaccination and Long-lasting Persistence of Effector Memory}

We constructed overlays of biomarker signatures of $\mathrm{NV}(\mathrm{d} 0)$ versus $\mathrm{PV}(\mathrm{d} 30-45)$ in the primary-vaccination arm of the study as well as \#PV $(y \geq 10)$ versus $R V(d 30-45)$ in the secondary-vaccination arm to select those attributes eligible as universal memory-related biomarkers (Appendix Figure 1, https://wwwnc.cdc.gov/EID/article/25/8/181432-App1.pdf). Venn diagram analysis revealed 3 common attributes (EMCD4, EMCD8, and IFNCD8) that we tagged for follow-up analysis after primary, secondary, or multiple 17DD-YF vaccination (Appendix Figure 1).

After we selected the universal set of follow-up attributes, we compared biomarker signatures over time after 17DD-YF primary or secondary vaccination (Figure 5). Data analysis demonstrated that all 3 biomarkers were observed in $\mathrm{PV}(\mathrm{d} 30-45)$ and $\mathrm{PV}(\mathrm{y} 1-5)$. EMCD8 was maintained in $P V(y>5-9)$ but not in $P V(y \geq 10)$. Comparative analysis between \#PV $(y \geq 10)$ and $\mathrm{RV}(\mathrm{d} 30-45)$ demonstrated restoration of universal memory-related biomarkers (EMCD4, EMCD8, and IFNCD8) upon revaccination. Moreover, we identified EMCD8 in a high proportion of secondary vaccinees across the time periods after booster dose (Figure 5).

\section{Comparison of 17DD-YF Primary and Booster Vaccination Effects on Cell-Mediated Memory}

We used individual PRNT and EMCD8 profiles to assemble a memory matrix and calculated the resultant YFspecific memory. We classified each volunteer by positive results above the cutoff threshold as EMCD8, PRNT, none, or both (Figure 6). Data analysis demonstrated that primary vaccination leads to a resultant memory in $97 \%$ of volunteers, with $3 \%$ of failure in the $\mathrm{PV}(\mathrm{d} 30-45)$ group. However, an increase in the proportion of primary vaccinees with "none" positive attributes, neither PRNT nor EMCD8 biomarkers, was observed, reaching a critical value of $30 \%$ at $\geq 10$ years after primary vaccination (Figure 6 , panel A).

The comparison between \#PV $(y \geq 10)$ and $\mathrm{RV}(\mathrm{d} 30-45)$ demonstrated that secondary vaccination restored the resultant memory in $100 \%$ of the volunteers, which was different from primary vaccination results. All secondary vaccines evaluated simultaneously for PRNT and EMCD8 profile presented a preserved resultant memory. In particular, at $\geq 10$ years after secondary vaccination, $100 \%$ of vaccinees had 1 or both biomarkers detectable (Figure 6, panel B).

\section{Multiple Vaccination and the Overall Profile of Protection}

We analyzed 17DD-YF memory status triggered by multiple vaccinations (Figure 7). The results demonstrated that 
all vaccinees who received multiple shots had PRNT levels above the cutoff limit (Figure 7, panel A). The proportion of vaccinees with EMCD8 above the cutoff limit was higher for $\mathrm{RV}(\mathrm{y} 1-5)$ and $\mathrm{RV} 2+(\mathrm{y} 1-5)$ than for $\mathrm{PV}(\mathrm{y} 1-5)$ (Figure 7, panel B). Likewise, the overall resultant memory for $\mathrm{RV}(\mathrm{y} 1-5)$ and $\mathrm{RV} 2+(\mathrm{y} 1-5)$ was higher than that for $\mathrm{PV}(\mathrm{y} 1-5)-($ Figure 7 , panel C).

\section{PRNT Seronegativity before Revaccination} and Cell-Mediated Memory Response

The volunteers from the \#PV $(y \geq 10)$ group were further categorized into subgroups, PRNT- and PRNT+, according to their PRNT results before revaccination. The levels of humoral and cellular biomarkers, as well as the magnitude of baseline fold changes in neutralizing antibodies, were higher in PRNT- than in PRNT+ vaccines (Figures 8, 9).
An increase in PRNT titer by a factor $>4$ at follow-up further demonstrated the relevance of booster doses to restore the immunological memory of these subjects (Figure 8). Furthermore, the booster dose had higher impact on cellular immunity and biomarker signature of PRNT- than PRNT+ primary vaccinees, and both groups restored the EMCD8 biomarker compared with the \#PV $(\mathrm{y} \geq 10)$ group (Figure 9).

\section{Dengue Virus Seropositivity}

We analyzed the results to determine whether DENV seropositivity influences the humoral and cellular memory after secondary vaccination (Appendix Figures 2, 3). We found DENV seropositivity in $28 \%$ of volunteers in the secondary vaccination arm. The results demonstrated that DENV seropositivity did not influence PRNT seropositivity

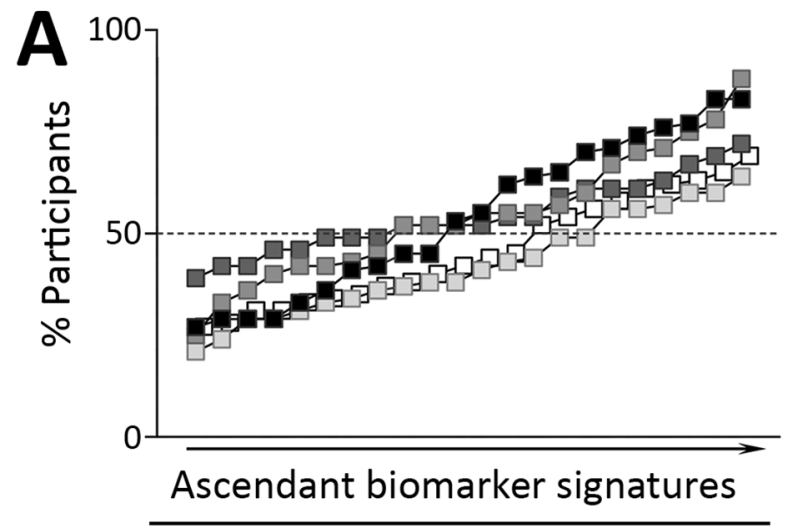

Time before/after primary vaccination

\begin{tabular}{|c|c|c|c|c|}
\hline$N V(d 0)$ & $P V(d 30-45)$ & $\operatorname{PV}(y 1-5)$ & $P V(y>5-9)$ & $P V(y \geq 10)$ \\
\hline IFNCD4 & CMCD8 & IL10CD4 & eEfCD4 & EMCD4 \\
\hline EMCD8 & CMCD4 & NCD4 & CMCD8 & EMCD8 \\
\hline CMCD4 & NCD8 & IL10CD8 & IFNCD8 & IFNCD4 \\
\hline TNFCD8 & IL10CD19 & IL5CD19 & IFNCD4 & eEfCD8 \\
\hline EMCD4 & NCD4 & CMCD8 & TNFCD4 & eEfCD4 \\
\hline TNFCD4 & IL10CD4 & TNFCD8 & TNFCD19 & CMCD19 \\
\hline eEfCD4 & IFNCD4 & NCD19 & EMCD4 & CMCD4 \\
\hline IL5CD4 & eEfCD4 & IL10CD19 & TNFCD8 & TNFCD4 \\
\hline NCD4 & IL10CD8 & eEfCD4 & eEfCD8 & CMCD8 \\
\hline IFNCD8 & TNFCD4 & EMCD4 & IL10CD8 & IL10CD8 \\
\hline IL10CD19 & eEfCD8 & TNFCD4 & IL5CD19 & TNFCD19 \\
\hline IL5CD8 & EMCD4 & EMCD8 & CMCD19 & NCD19 \\
\hline CMCD19 & EMCD8 & CMCD4 & IL10CD4 & IFNCD8 \\
\hline IL5CD19 & TNFCD8 & IL5CD4 & IL5CD4 & $\overline{\text { TNFCD8 }}$ \\
\hline TNFCD19 & IFNCD8 & TNFCD19 & EMCD8 & IL10CD4 \\
\hline CD8IL10 & NCD19 & IFNCD4 & NCD19 & IL5CD4 \\
\hline NCD8 & TNFCD19 & eEfCD8 & IL10CD19 & IL5CD8 \\
\hline CD4IL10 & IL5CD8 & IL5CD8 & nCMCD19 & IL5CD19 \\
\hline NCD19 & CMCD19 & CMCD19 & NCD8 & IL10CD19 \\
\hline CMCD8 & IL5CD19 & nCMCD19 & CMCD4 & NCD4 \\
\hline eEfCD8 & nCMCD19 & IFNCD 8 & IL5CD8 & NCD8 \\
\hline nCMCD19 & IL5CD4 & NCD8 & NCD4 & nCMCD19 \\
\hline
\end{tabular}

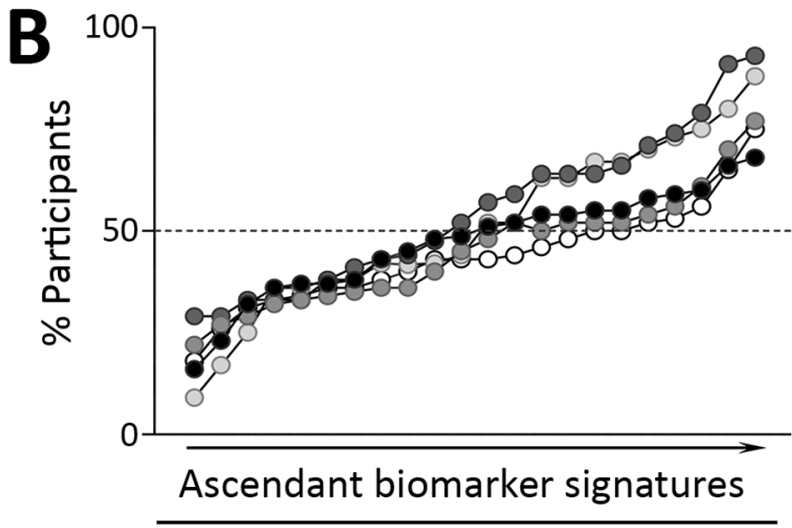

Time before/after secondary vaccination

\begin{tabular}{|c|c|c|c|c|}
\hline$\# P V(y \geq 10)$ & $\mathrm{RV}(\mathrm{d} 30-45)$ & $R V(y 1-5)$ & $R V(y>5-9)$ & $R V(y \geq 10)$ \\
\hline eEfCD8 & nCMCD19 & NCD4 & IFNCD8 & nCMCD19 \\
\hline IL5CD8 & NCD19 & CMCD19 & IL10CD19 & EMCD4 \\
\hline eEfCD4 & IL10CD19 & nCMCD19 & IL5CD19 & NCD8 \\
\hline IL5CD4 & NCD8 & IL5CD8 & TNFCD19 & NCD19 \\
\hline IL5CD19 & CMCD8 & IL10CD19 & NCD8 & CMCD19 \\
\hline nCMCD19 & TNFCD8 & NCD8 & IL5CD8 & NCD4 \\
\hline NCD8 & IL5CD8 & TNFCD19 & IL5CD4 & eEfCD8 \\
\hline NCD19 & IL10CD8 & IL5CD19 & NCD19 & IL10CD19 \\
\hline EMCD4 & IL5CD4 & IL5CD4 & CMCD19 & IL10CD4 \\
\hline CD4IL10 & NCD4 & IL10CD4 & IL10CD8 & IL5CD19 \\
\hline TNFCD19 & IL10CD4 & IL10CD8 & nCMCD19 & TNFCD19 \\
\hline TNFCD8 & eEfCD4 & IFNCD8 & NCD4 & CMCD8 \\
\hline EMCD8 & CMCD19 & TNFCD8 & IL10CD4 & IFNCD8 \\
\hline CMCD19 & eEfCD8 & CMCD4 & IFNCD4 & CMCD4 \\
\hline TNFCD4 & IFNCD8 & CMCD8 & TNFCD8 & EMCD8 \\
\hline IFNCD8 & IFNCD4 & NCD19 & CMCD4 & TNFCD4 \\
\hline IL10CD19 & IL5CD19 & TNFCD4 & eEfCD8 & TNFCD8 \\
\hline NCD4 & TNFCD4 & eEfCD8 & TNFCD4 & IFNCD4 \\
\hline IFNCD4 & EMCD8 & IFNCD4 & EMCD4 & IL5CD4 \\
\hline CD8IL10 & CMCD4 & EMCD8 & EMCD8 & IL10CD8 \\
\hline CMCD8 & EMCD4 & EMCD4 & CMCD8 & IL5CD8 \\
\hline CMCD4 & TNFCD19 & eEfCD4 & eEfCD4 & eEfCD4 \\
\hline
\end{tabular}

Figure 5. Ascendant biomarker signatures before and after primary and secondary 17DD vaccination for YF. Overlaid biomarker signatures were assembled to identify changes in the 17DD-specific phenotypic and functional features observed over time in primary vaccination arm (A) and secondary vaccination arm (B). Shading indicates time point before and after primary or secondary vaccination for each biomarker; dashed line indicates the global median $>50$ th percentile. Boldface text indicates the 3 biomarkers considered relevant universal attributes to monitor 17DD-YF-specific memory (EMCD4, EMCD8, IFNCD8). Participant subgroups indicate number of days or years since vaccination (in parentheses; d0 for those never vaccinated). IFN, interferon; IL, interleukin; TNF, tumor necrosis factor; YF, yellow fever. 
A

$\mathrm{NV}(\mathrm{d} 0)$
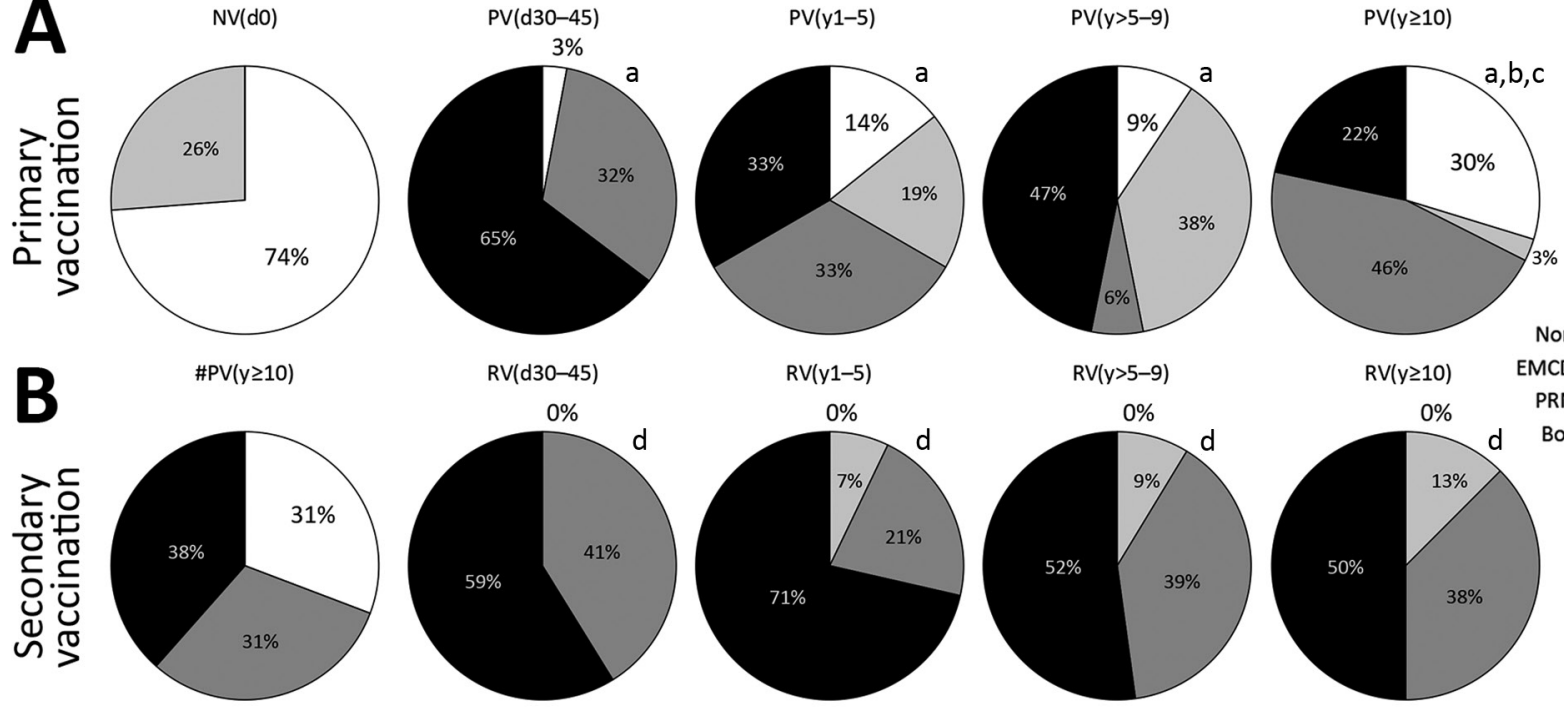

Figure 6. Overall proportion of participants with resultant memory before and after primary or secondary 17DD vaccination for YF as assessed by PRNT and EMCD8 measurement at participant level. Graphs show the proportion of participants above the cutoff threshold; that is, PRNT positivity at serum dilution >1:50 and EMCD8 index above the global median value. We then determined the resultant memory for each subgroup in the primary vaccination arm (A) and secondary vaccination (B) of the study. Participant subgroups indicate number of days or years since vaccination (in parentheses; d0 for those never vaccinated). Lowercase letters indicate significant differences ( $p<0.05$ by $\chi^{2}$ test) of resultant memory status among study groups: a for comparisons with $\mathrm{NV}(\mathrm{d} 0)$ participants; b for comparisons with $\mathrm{PV}(\mathrm{d} 30-45)$ participants; c for comparison with $\mathrm{PV}(\mathrm{y} 1-5)$ participants; and d for comparison with \#PV $(y \geq 10)$ participants. \#PV, had primary vaccination $\geq 10$ years previously; NV, not vaccinated; PRNT, plaque-reducing neutralization test; $\mathrm{PV}$, had primary vaccination only; RV, revaccinated; YF, yellow fever.

over time after secondary vaccination (Appendix Figure 2). Moreover, DENV seropositivity did not affect cellular immunity (EMCD8), which remained above the cutoff limit in all subgroups of secondary vaccinees (Appendix Figure 3).

\section{Discussion}

YF vaccination induces an efficient immunity and represents one of the most effective strategies to reduce the risk for infection in YF-endemic countries. The vaccine is highly immunogenic, eliciting a strong antibody response together with a broad and complex innate (16-22) and adaptive immunity (23-28). Although the YF vaccine has been considered a benchmark among vaccines because of its ability to induce long-lasting immune response, the duration of humoral and cellular immunity following YF vaccination is still controversial. Some studies demonstrate that neutralizing antibodies and YF-specific CD8+ T cells after primary vaccination are suggestive imprints compatible with long-lived memory $(8,26,29-31)$; other studies emphasize that the immunity to YF vaccine wanes over time, suggesting the need for booster doses to guarantee long-lasting, efficient immunity memory (9-12,32-36).

We could not assess protective immunity to YF in humans by challenge with live wild-type YF virus. Therefore, the protective or nonprotective immunity status to YF virus in humans is based on laboratory methods, known as correlates of protection; PRNT level has been considered the standard for measuring postvaccination immunity to YF. Pinpointing cellular immunity biomarkers is relevant in studies that pose the question of whether PRNT seronegativity necessarily means absence of protective immunity (34). Our investigation proposes that, in addition to PRNT seropositivity, YF-specific cellular immunity may be a useful tool for monitoring the duration of YF vaccine-induced memory over time. However, upon closer examination, our findings indicate the decline of YF-specific immune response over time, emphasizing that the YF-specific immunity wanes shortly after primary vaccination and that a substantial proportion of primary vaccines $(14 \%-30 \%)$ do not present sufficient levels of neutralizing antibodies or CD8+ T-cell memory within 5-10 years after primary vaccination.

Some studies have investigated the relevance of booster doses to heighten the YF-specific immune response in primary vaccinees whose immunity has declined to nonprotective levels. Weiten et al. (31) postulated that booster vaccination did not increase the titers of YF-specific antibodies nor induce or alter the phenotypes of CD8+ T cells. Conversely, Kongsgaard et al. (37) have demonstrated that, although most vaccinees responded to a booster vaccination, the antibody titers and the cellular immune responses observed following revaccination were lower than for primary vaccination responses. In this study, we have demonstrated that the booster dose was able to upregulate the 
levels of neutralizing antibodies and heighten the cellular immunity signature and restore the proportion of vaccinated participants with high levels of effector memory CD8+ T cells. We strongly believe that the differences we observed among the published studies could be attributed to differences in the populations under scrutiny.

The magnitude of increase in the neutralizing antibody titers and YF-specific CD8+ T-cell response achieved with a booster dose may be closely related to the baseline immune activation, suggesting that an activated immune microenvironment before revaccination impairs the response to the YF vaccine. Muyanja et al.
(38) showed that 17D-204 vaccinees in Africa displayed decreased levels of YF-specific immunity compared with vaccinees in Europe. It is possible that the immune microenvironment affects the quantitative and qualitative response, as well as the vaccine efficacy. Whereas vaccinees in Europe displayed persistent PRNT levels even at 10 years after vaccination, those in Africa exhibited reduced persistence of YF immunological memory. Constant exposure to infectious diseases, as well as diet and gut microbiota, may lead to a state of immune hyperactivation and exhaustion that can contribute to reduced magnitude of cellular and humoral responses and impair the YF
A

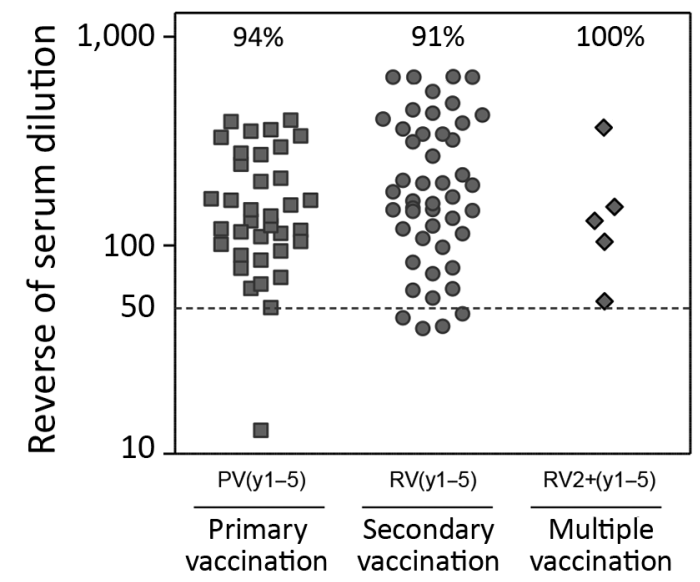

C

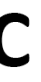

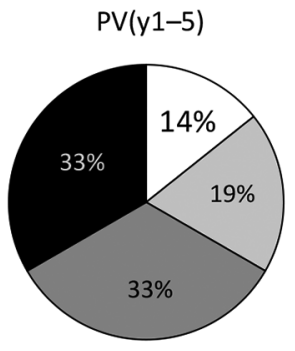

$\mathrm{RV} 2+(\mathrm{y} 1-5)$

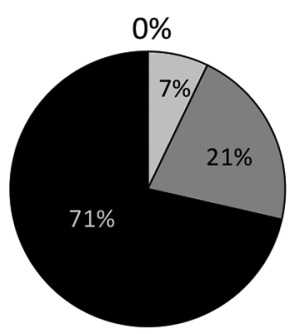

B

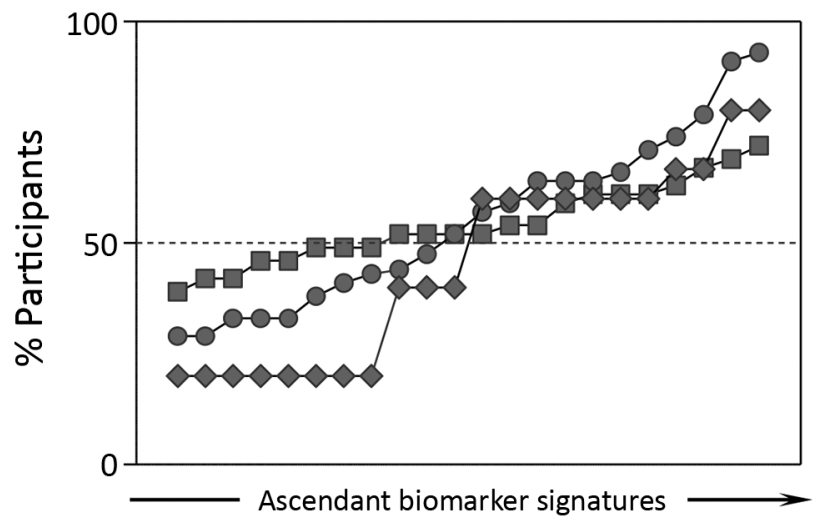

\begin{tabular}{ccc}
\hline PV(y1-5) & RV(y1-5) & RV2+(y1-5) \\
\hline IL10CD4 & NCD4 & NCD19 \\
NCD4 & CMCD19 & IFNCD8 \\
IL10CD8 & nCMCD19 & EMCD4 \\
IL5CD19 & IL5CD8 & eEfCD8 \\
CMCD8 & IL10CD19 & IFNCD4 \\
TNFCD8 & NCD8 & IL5CD4 \\
NCD19 & TNFCD19 & TNFCD19 \\
IL10CD19 & IL5CD19 & IL5CD19 \\
\hline eEfCD4 & IL5CD4 & CMCD4 \\
EMCD4 & IL10CD4 & IL10CD8 \\
\hline TNFCD4 & IL10CD8 & IL10CD8 \\
\hline EMCD8 & IFNCD8 & NCD4 \\
\hline CMCD4 & TNFCD8 & NCD8 \\
IL5CD4 5 TNCDCD4 & CMCD4 & TNFCD4 \\
TNFCD19 & CMCD8 & TNFCD8 \\
IFNCD4 & NCD19 & IL5CD8 \\
eEfCD8 & TNFCD4 & IL10CD19 \\
IL5CD8 & eEfCD8 & EMCD8 \\
\hline CMCD19 & IFNCD4 & nCMCD19 \\
\hline nCMCD19 & EMCD8 & CMCD19 \\
IFNCD8 & EMCD4 & EEfCD4 \\
\hline NCD8 & eEfCD4 & CMCD8 \\
\hline
\end{tabular}

Figure 7. Overall profile of immune response after primary, secondary, or multiple 17DD vaccination for YF. A) Levels of 17DD-YFspecific neutralizing antibodies; B) 17DD-YF-specific phenotypic and functional biomarkers; and C) resultant memory status PRNT and EMCD8 measurement (PRNT and EMCD8) for individual participants. Results are expressed in reverse of serum dilution, percentage of participants with overlaid biomarker signatures, and resultant memory status at 1-5 years after primary (gray circle), secondary (gray square), or multiple (gray diamond) vaccination. Participant subgroups indicate number of days or years since vaccination (in parentheses; d0 for those never vaccinated). \#PV, had primary vaccination $\geq 10$ years previously; IFN, interferon; IL, interleukin; NV, not vaccinated; PRNT, plaque-reducing neutralization test; PV, had primary vaccination only; RV, revaccinated; TNF, tumor necrosis factor; YF, yellow fever. 

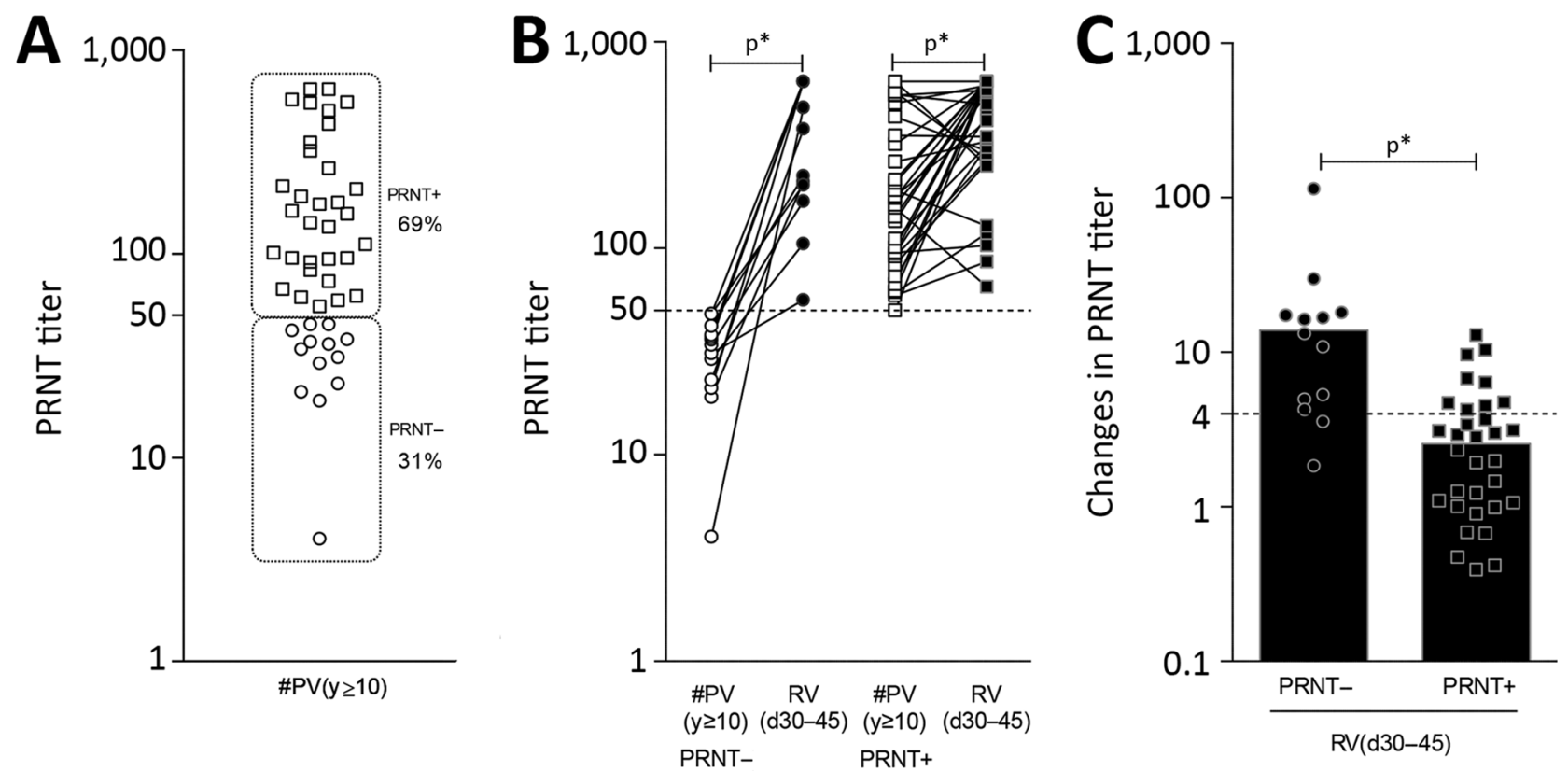

Figure 8. Baseline PRNT reactivity before revaccination $(A)$ and impact on PRNT levels triggered by secondary 17DD vaccination for YF (B, C). Subgroups PRNT- (circles) and PRNT+ (squares) were defined considering the cutoff criterion for PRNT positivity at serum dilution $>1: 50$. The ability of secondary vaccination to increase the levels of neutralizing antibodies as well as the magnitude of changes in PRNT titers (baseline fold changes) are indicated for PRNT- (filled circles) and PRNT+ (filled squares) vaccinees. Increases in PRNT titer by a factor of $\geq 4$ at follow-up were considered as classical criteria to evaluate booster response. Bars indicate significant differences $(p<0.05)$ between subgroups. Participant subgroups indicate number of days or years since vaccination (in parentheses). \#PV, had primary vaccination $\geq 10$ years previously; PRNT, plaque-reducing neutralization test; RV, revaccinated; YF, yellow fever.

vaccine memory. Of note, the impaired YF vaccine-induced memory was boosted by a second vaccination (38). Our results corroborate this hypothesis, showing the relevance of a booster dose to improve the immune response among primary vaccinees. This recommendation should be considered to restore the YF protective immunity in those primary vaccinees whose correlates of protection fade over time, reaching nonprotective levels.

The loss of immunity in a subpopulation of vaccine recipients should be taken into consideration, and a booster dose should be administered. Previous studies have demonstrated that, on average, approximately 1 in 5 persons from non-YF-endemic areas and 3 in 10 persons living in YF-endemic areas may lose measurable antibody responses within 10 years after primary vaccination. In this sense, it is true that the 17DD-YF vaccination elicits long-lasting immunity; however, lifelong immunity is not observed in every vaccinated individual. Although PRNT is the standard assay to monitor YF protective immunity, it is not a feasible method available in local laboratories to identify participants who should receive a booster vaccination.

The expansion of risk areas for YF worldwide has contributed to the depletion of YF vaccine stockpile and the need for new strategies to reverse the imminent shortage of vaccine. WHO has considered measures to improve YF vaccine supply, including a dose-sparing strategy as a shortterm measure. However, the dose sparing is not proposed for routine immunization. WHO also considered advising a single lifetime dose of YF vaccine. In YF outbreaks or periods of limited vaccine production and reduced stockpiles, the YF primary vaccination should be the priority, as recommended by the Strategic Advisory Group of Experts on Immunization. However, once the YF vaccine supply has normalized and no outbreaks are reported, revaccination should be suggested. On the basis of our findings, $\geq 1$ booster dose at 10 years after primary vaccination is suggested for travelers entering higher-risk areas and required for residents of YF-endemic countries who are at risk for infection $(34,39)$.

Altogether, our findings emphasize the relevance of booster doses to heighten the 17DD-YF specific immune response to achieve efficient immunity. Secondary vaccination improved the correlates of protection that had waned over time after 17DD-YF primary vaccination and lowered the loss of protection over time. However, multiple vaccination seems to better support long-lasting protection in all vaccinees, which suggests that $\geq 3$ booster doses at 10-year intervals should be recommended, especially in areas with high risk for YF transmission. Whether the waning of immune markers observed over time is associated with loss of YF vaccine effectiveness 


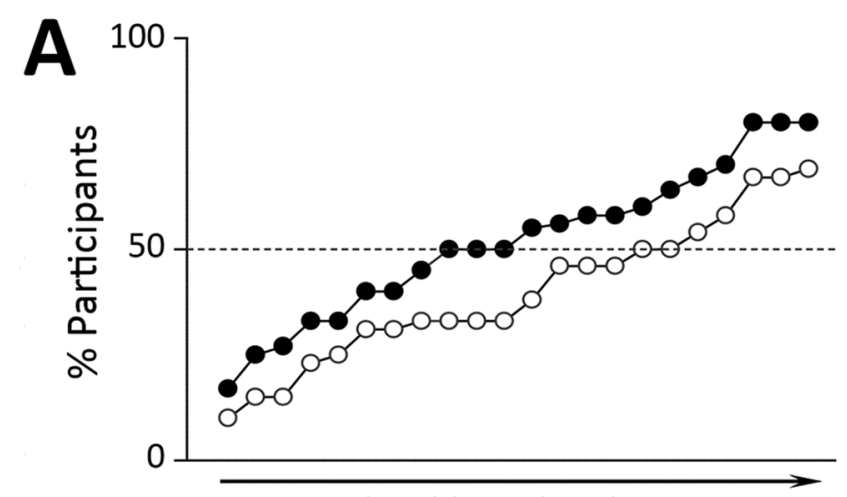

Ascendant biomarker signatures

Time after vaccination

\begin{tabular}{|c|c|}
\hline$\# P V(y \geq 10)$ & $R V(d 30-45)$ \\
\hline eEfCD8 & nCMCD19 \\
\hline IL5CD8 & NCD19 \\
\hline IL10CD19 & TNFCD8 \\
\hline IL5CD19 & NCD8 \\
\hline TNFCD19 & CMCD8 \\
\hline IL10CD4 & TNFCD19 \\
\hline IL5CD4 & IL10CD19 \\
\hline eEfCD4 & IL5CD4 \\
\hline EMCD4 & NCD4 \\
\hline CMCD8 & CMCD19 \\
\hline EMCD8 & IL5CD8 \\
\hline CMCD19 & IFNCD4 \\
\hline nCMCD19 & TNFCD4 \\
\hline IFNCD4 & CMCD4 \\
\hline IL10CD8 & eEfCD8 \\
\hline IFNCD8 & IL10CD4 \\
\hline CMCD4 & IL10CD8 \\
\hline NCD19 & EMCD4 \\
\hline TNFCD8 & IL5CD19 \\
\hline NCD4 & eEfCD4 \\
\hline NCD8 & EMCD8 \\
\hline TNFCD4 & IFNCD8 \\
\hline
\end{tabular}

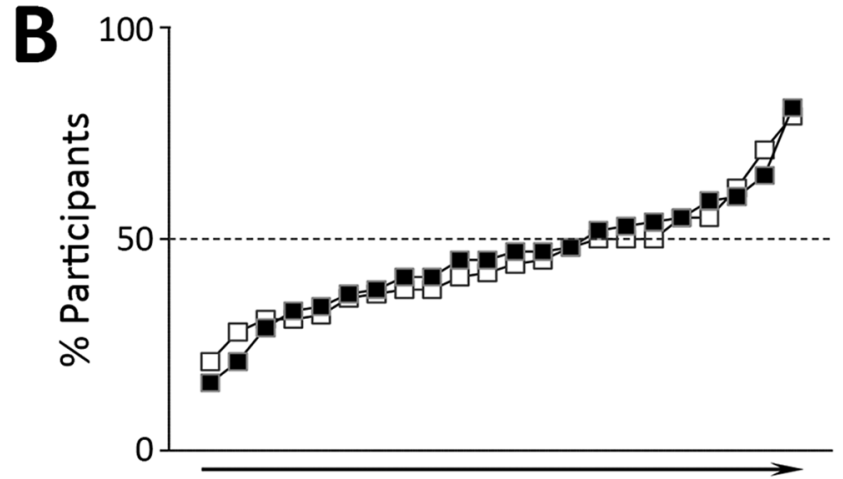

Ascendant biomarker signatures

Time after vaccination

\begin{tabular}{|c|c|}
\hline$\# P V(y \geq 10)$ & $\operatorname{RV}(d 30-45)$ \\
\hline eEfCD8 & nCMCD19 \\
\hline NCD19 & NCD19 \\
\hline NCD8 & IL10CD19 \\
\hline IL10CD19 & IL5CD8 \\
\hline nCMCD19 & IL10CD8 \\
\hline IL5CD4 & NCD8 \\
\hline IL5CD19 & CMCD8 \\
\hline IL5CD8 & eEfCD4 \\
\hline TNFCD8 & TNFCD8 \\
\hline TNFCD4 & IL10CD4 \\
\hline eEfCD4 & IL5CD4 \\
\hline IFNCD8 & NCD4 \\
\hline EMCD4 & IL5CD19 \\
\hline IL10CD4 & IFNCD8 \\
\hline NCD4 & $\overline{\text { eEfCD8 }}$ \\
\hline EMCD8 & CMCD19 \\
\hline TNFCD19 & EMCD8 \\
\hline CMCD19 & IFNCD4 \\
\hline IL10CD8 & TNFCD4 \\
\hline IFNCD4 & CMCD4 \\
\hline CMCD8 & EMCD4 \\
\hline CMCD4 & TNFCD19 \\
\hline
\end{tabular}

Figure 9. Impact of baseline plaque-reducing neutralization test (PRNT) reactivity on memory-related biomarkers triggered by secondary 17DD vaccination for YF. We assessed the impact of secondary vaccination on phenotypic and functional biomarkers in participants with negative (A) and positive (B) PRNT results. Shading indicates time point after primary vaccination for each biomarker: white for participants who were vaccinated $\geq 10$ years previously, and black for those who were revaccinated in the previous $30-45$ days. The dashed line shows the global median $>50$ th percentile. Boldface text indicates the 3 biomarkers considered relevant universal attributes to monitor 17DD-YFspecific memory (EMCD4, EMCD8, IFNCD8). Participant subgroups indicate number of days or years since vaccination (in parentheses). \#PV, had primary vaccination $\geq 10$ years previously; IFN, interferon; IL, interleukin; RV, revaccinated; TNF, tumor necrosis factor; YF, yellow fever.

and increased risk for breakthrough YF infection remains to be seen.

In summary, we evaluated the evidence for benefits and risks associated with YF vaccine booster doses using the Grading of Recommendations, Assessment, Development, and Evaluation framework (40). A primary dose of YF vaccine is well known to be highly safe and effective, with few vaccine failures. However, a critical wane of correlates of protection has been documented by our group and others, particularly at $\geq 10$ years postvaccination. Few reports of serious adverse events have been observed following booster doses of the vaccine. We recommend the booster dose to prevent this serious disease that has no treatment and substantial mortality rates.

\section{Acknowledgments}

We thank the Program for Technological Development in Tools for Health-RPT-FIOCRUZ for the use of the flow cytometry facility.

This study was supported by Fundação de Amparo à Pesquisa do Estado de Minas Gerais, Coordenação de Aperfeiçoamento de Pessoal de Nível Superior, Bio-Manguinhos/FIOCRUZ, PROEP/ CPqRR/FIOCRUZ, Conselho Nacional de Desenvolvimento Científico e Tecnológico-CNPq (grant nos. 444417/2014-1 and 458134/2014-7), and Programa Nacional de Imunizações, Secretaria de Vigilância em Saúde-Ministério da Saúde, Brazil (grant no. TC 277/2013). O.A.M.-F., A.T.-C., and L.R.A. received research fellowships from $\mathrm{CNPq}$. 
Author contributions: study design, A.C.C.-A., L.A.B.C., M.L.S.M., R.M.M., A.H., P.L.T., P.F.C.V., A.P.M.R., C.M.D., A.T.-C., O.A.M.-F.; funding acquisition, L.A.B.C., O.A.M.-F., A.P.M.R., C.M.D.; experiments: A.C.C.-A., V.P.-M., J.G.C.-R., L.R.A., C.C.-P., E.S., L.R.R., S.M.B.L., M.S., A.T.-C., O.A.M.-F.; field study: J.A.L., J.G.L.R., L.A.B.C., L.C.C.M.; data acquisition: A.C.C.-A., V.P.-M., J.G.C.-R., L.R.A., C.C.-P., E.S., S.M.B.L., M.S.; data analysis: A.C.C.-A., A.T.-C., O.A.M.-F.; validation: P.L.T., P.F.C.V.; advisory committee: R.M.M., A.H., P.L.T., P.F.C.V.; manuscript: A.C.C.-A., J.G.C.-R., C.C.-P., L.R.R., A.T.-C., O.A.M.-F.

\section{About the Author}

Dr. Campi-Azevedo is a cellular and molecular immunologist working in the Biomarker Research Group at the René Rachou Institute, Oswaldo Cruz Foundation (FIOCRUZ-Minas), Belo Horizonte, Brazil. Her primary research interests include vaccine immunity; diagnosis and management of infectious/parasitic diseases and biomarkers for posttherapeutic follow-up; bioprospection in experimental therapeutics; and biomarkers in oncology.

\section{References}

1. World Health Organization. Vaccines and vaccination against yellow fever. WHO position paper-June 2013. Wkly Epidemiol Rec. 2013;88:269-83.

2. World Health Organization. Yellow fever in Disease Outbreak News [cited 2019 Jun 20]. https://www.who.int/csr/don/archive/ disease/yellow_fever

3. The Lancet. Yellow fever: a major threat to public health. Lancet. 2018;391:402. http://dx.doi.org/10.1016/S0140-6736(18)30152-1

4. de Almeida MA, Dos Santos E, da Cruz Cardoso J, da Fonseca DF, Noll CA, Silveira VR, et al. Yellow fever outbreak affecting Alouatta populations in southern Brazil (Rio Grande do Sul State), 2008-2009. Am J Primatol. 2012;74:68-76. http://dx.doi.org/10.1002/ajp.21010

5. Romano APM, Costa ZGA, Ramos DG, Andrade MA, Jayme VdS, Almeida MA, et al. Yellow fever outbreaks in unvaccinated populations, Brazil, 2008-2009. PLoS Negl Trop Dis. 2014;8:e2740.

6. Secretaria de Vigilância em Saúde-Ministério da Saúde. Reemergence of wild yellow fever in Brazil, 2014-2015: epidemiological situation and the importance of preventive vaccination and intensified surveillance in the seasonal period [in Portuguese]. Boletim Epidemiológico. 2015;46:29 [cited 2019 May 29]. http://portalarquivos2.saude.gov.br/images/pdf/2015/ outubro/19/2015-032---FA-ok.pdf

7. Secretaria de Vigilância em Saúde-Ministério da Saúde. Epidemiological emergence of yellow fever in Brazil, December 2016-July 2017 [in Portuguese]. Boletim Epidemiológico. 2017; 48:28 [cited 2019 May 29]. http://portalarquivos2.saude.gov.br/ images/pdf/2017/setembro/06/2017_027.pdf

8. World Health Organization. Background paper on yellow fever vaccine. In: Vaccine position papers. Geneva: SAGE Working Group; 2013.

9. Collaborative group for studies on yellow fever vaccines. Duration of post-vaccination immunity against yellow fever in adults. Vaccine. 2014;32:4977-84. http://dx.doi.org/10.1016/ j.vaccine.2014.07.021
10. Niedrig M, Lademann M, Emmerich P, Lafrenz M. Assessment of IgG antibodies against yellow fever virus after vaccination with 17D by different assays: neutralization test, haemagglutination inhibition test, immunofluorescence assay and ELISA. Trop Med Int Health. 1999;4:867-71. http://dx.doi.org/10.1046/ j.1365-3156.1999.00496.x

11. Campi-Azevedo AC, Costa-Pereira C, Antonelli LR, Fonseca CT, Teixeira-Carvalho A, Villela-Rezende G, et al. Booster dose after 10 years is recommended following 17DD-YF primary vaccination. Hum Vaccin Immunother. 2016;12:491-502. http://dx.doi.org/ $10.1080 / 21645515.2015 .1082693$

12. Costa-Pereira C, Campi-Azevedo AC, Coelho-dos-Reis JG, Peruhype-Magalhães V, Araújo MSS, do Vale Antonelli LR, et al. Multi-parameter approach to evaluate the timing of memory status after 17DD-YF primary vaccination. PLoS Negl Trop Dis. 2018;12:e0006462. http://dx.doi.org/10.1371/journal.pntd.0006462

13. Simões M, Camacho LAB, Yamamura AMY, Miranda EH, Cajaraville ACRA, da Silva Freire M. Evaluation of accuracy and reliability of the plaque reduction neutralization test (micro-PRNT) in detection of yellow fever virus antibodies. Biologicals. 2012;40:399-404. http://dx.doi.org/10.1016/ j.biologicals.2012.09.005

14. Nogueira RMR. Diagnóstico Laboratorial Específico e Diagnóstico Imunológico da Dengue [Specific laboratory diagnosis and immunodiagnosis of dengue]. Dengue Diagnóstico, Tratamento e Prevenção [Dengue diagnosis, treatment and prevention]. 2nd ed. Rio de Janeiro (Brazil): Editora Rubio Ltda.; 2008. p. 97-108.

15. Luiza-Silva M, Campi-Azevedo AC, Batista MA, Martins MA, Avelar RS, da Silveira Lemos D, et al. Cytokine signatures of innate and adaptive immunity in 17DD yellow fever vaccinated children and its association with the level of neutralizing antibody. J Infect Dis. 2011;204:873-83. http://dx.doi.org/10.1093/infdis/ jir439

16. Querec T, Bennouna S, Alkan S, Laouar Y, Gorden K, Flavell R, et al. Yellow fever vaccine YF-17D activates multiple dendritic cell subsets via TLR2, 7, 8, and 9 to stimulate polyvalent immunity. J Exp Med. 2006;203:413-24. http://dx.doi.org/10.1084/ jem.20051720

17. Querec TD, Akondy RS, Lee EK, Cao W, Nakaya HI, Teuwen D, et al. Systems biology approach predicts immunogenicity of the yellow fever vaccine in humans. Nat Immunol. 2009;10:116-25. http://dx.doi.org/10.1038/ni.1688

18. Martins MA, Silva ML, Marciano APV, Peruhype-Magalhães V, Eloi-Santos SM, Ribeiro JGL, et al. Activation/modulation of adaptive immunity emerges simultaneously after 17DD yellow fever first-time vaccination: is this the key to prevent severe adverse reactions following immunization? Clin Exp Immunol. 2007;148:90-100. http://dx.doi.org/10.1111/ j.1365-2249.2006.03317.x

19. Martins MA, Silva ML, Elói-Santos SM, Ribeiro JG, Peruhype-Magalhães V, Marciano AP, et al. Innate immunity phenotypic features point toward simultaneous raise of activation and modulation events following 17DD live attenuated yellow fever first-time vaccination. Vaccine. 2008;26:1173-84. http://dx.doi.org/10.1016/j.vaccine.2007.12.035

20. Pulendran B. Learning immunology from the yellow fever vaccine: innate immunity to systems vaccinology. Nat Rev Immunol. 2009;9:741-7. http://dx.doi.org/10.1038/nri2629

21. Silva ML, Martins MA, Espírito-Santo LR, Campi-Azevedo AC, Silveira-Lemos D, Ribeiro JG, et al. Characterization of main cytokine sources from the innate and adaptive immune responses following primary 17DD yellow fever vaccination in adults. Vaccine. 2011;29:583-92. http://dx.doi.org/10.1016/j.vaccine. 2010.08.046

22. Kohler S, Bethke N, Böthe M, Sommerick S, Frentsch M, Romagnani $\mathrm{C}$, et al. The early cellular signatures of protective 
immunity induced by live viral vaccination. Eur J Immunol. 2012;42:2363-73. http://dx.doi.org/10.1002/eji.201142306

23. Miller JD, van der Most RG, Akondy RS, Glidewell JT, Albott S, Masopust D, et al. Human effector and memory CD8+ T cell responses to smallpox and yellow fever vaccines. Immunity. 2008;28:710-22. http://dx.doi.org/10.1016/j.immuni.2008.02.020

24. Akondy RS, Monson ND, Miller JD, Edupuganti S, Teuwen D, $\mathrm{Wu} \mathrm{H}$, et al. The yellow fever virus vaccine induces a broad and polyfunctional human memory CD8+ T cell response. J Immunol. 2009;183:7919-30. http://dx.doi.org/10.4049/jimmunol.0803903

25. James EA, LaFond RE, Gates TJ, Mai DT, Malhotra U, Kwok WW. Yellow fever vaccination elicits broad functional CD4+ T-cell responses that recognize structural and nonstructural proteins. $\mathrm{J}$ Virol. 2013;87:12794-804. http://dx.doi.org/10.1128/JVI.01160-13

26. Reinhardt B, Jaspert R, Niedrig M, Kostner C, L'age-Stehr J. Development of viremia and humoral and cellular parameters of immune activation after vaccination with yellow fever virus strain 17D: a model of human flavivirus infection. J Med Virol. 1998;56:159-67. http://dx.doi.org/10.1002/(SICI) 1096-9071(199810)56:2<159::AID-JMV10>3.0.CO;2-B

27. Akondy RS, Fitch M, Edupuganti S, Yang S, Kissick HT, Li KW, et al. Origin and differentiation of human memory CD8 T cells after vaccination. Nature. 2017;552:362-7. http://dx.doi.org/10.1038/ nature 24633

28. Blom K, Braun M, Ivarsson MA, Gonzalez VD, Falconer K, Moll M, et al. Temporal dynamics of the primary human T-cell response to yellow fever virus $17 \mathrm{D}$ as it matures from an effector- to a memory-type response. J Immunol. 2013;190:2150-8. http://dx.doi.org/10.4049/jimmunol.1202234

29. Poland JD, Calisher CH, Monath TP, Downs WG, Murphy K. Persistence of neutralizing antibody $30-35$ years after immunization with $17 \mathrm{D}$ yellow fever vaccine. Bull World Health Organ. 1981;59:895-900.

30. Gotuzzo E, Yactayo S, Córdova E. Efficacy and duration of immunity after yellow fever vaccination: systematic review on the need for a booster every 10 years. Am J Trop Med Hyg. 2013;89:434-44. http://dx.doi.org/10.4269/ajtmh.13-0264

31. Wieten RW, Jonker EF, van Leeuwen EM, Remmerswaal EB, Ten Berge IJ, de Visser AW, et al. A single 17D yellow fever vaccination provides lifelong immunity; characterization of yellowfever-specific neutralizing antibody and T-cell responses after vaccination. PLoS One. 2016;11:e0149871.
32. Patel D, Simons H. Yellow fever vaccination: is one dose always enough? Travel Med Infect Dis. 2013;11:266-73. http://dx.doi.org/ 10.1016/j.tmaid.2013.08.007

33. Grobusch MP, Goorhuis A, Wieten RW, Verberk JD, Jonker EF, van Genderen PJ, et al. Yellow fever revaccination guidelines change - a decision too feverish? Clin Microbiol Infect. 2013; 19:885-6. http://dx.doi.org/10.1111/1469-0691.12332

34. Amanna IJ, Slifka MK. Questions regarding the safety and duration of immunity following live yellow fever vaccination. Expert Rev Vaccines. 2016;15:1519-33. http://dx.doi.org/10.1080/ 14760584.2016.1198259

35. Vasconcelos PF. Single shot of 17D vaccine may not confer life-long protection against yellow fever. Mem Inst Oswaldo Cruz. 2018;113:135-7. http://dx.doi.org/10.1590/0074-02760170347

36. Estofolete CF, Nogueira ML. Is a dose of $17 \mathrm{D}$ vaccine in the current context of yellow fever enough? Braz J Microbiol. 2018;S1517-8382(18)30196-5.

37. Kongsgaard M, Bassi MR, Rasmussen M, Skjødt K, Thybo S, Gabriel M, et al. Adaptive immune responses to booster vaccination against yellow fever virus are much reduced compared to those after primary vaccination. Sci Rep. 2017;7:662. http://dx.doi.org/10.1038/s41598-017-00798-1

38. Muyanja E, Ssemaganda A, Ngauv P, Cubas R, Perrin H, Srinivasan $\mathrm{D}$, et al. Immune activation alters cellular and humoral responses to yellow fever 17D vaccine. J Clin Invest. 2014;124:3147-58. http://dx.doi.org/10.1172/JCI75429

39. Staples JE, Bocchini JA Jr, Rubin L, Fischer M; Centers for Disease Control and Prevention (CDC). Yellow fever vaccine booster doses: recommendations of the Advisory Committee on Immunization Practices, 2015. MMWR Morb Mortal Wkly Rep. 2015;64:647-50.

40. Centers for Disease Control and Prevention (CDC). GRADE evidence tables. 2015 [cited 2019 May 24]. http://www.cdc.gov/ vaccines/acip/recs/GRADE/table-refs.html

Address for correspondence: Olindo Assis Martins-Filho or Ana Carolina Campi-Azevedo, Grupo Integrado de Pesquisas em Biomarcadores - Instituto René Rachou, FIOCRUZ-Minas, Avenida Augusto de Lima, 1715, Barro Preto, Belo Horizonte, Minas Gerais, 30.190-002, Brazil; email: oamfilho@minas.fiocruz.br or accampi@minas.fiocruz.br 